$\mathrm{TTP} / 00-11$

hep-ph/0005301

May 2000

\title{
Subleading Sudakov Logarithms in Electroweak Processes *
}

\author{
J.H. Kühn ${ }^{a}$, A.A. Penin ${ }^{\dagger},{ }^{b}$ and V.A. Smirnov ${ }^{c}$ \\ anstitut für Theoretische Teilchenphysik, Universität Karlsruhe, 76128 Karlsruhe, Germany \\ bII. Institut für Theoretische Physik, Universität Hamburg, 22761 Hamburg, Germany \\ cNuclear Physics Institute of Moscow State University, 119899 Moscow, Russia
}

Recent results for the asymptotic behavior of fermion scattering amplitudes in the Sudakov limit are presented including next-to-leading logarithmic corrections. These are used for the analysis of the dominant electroweak corrections to the fermion-antifermion pair production in $e^{+} e^{-}$annihilation at high energy.

\section{Introduction}

Four fermion processes are generally considered as benchmark processes at high energy colliders, with electron positron annihilation into muon or quark pairs at LEP and the Drell Yan process at hadron colliders as characteristic examples. Within the presently accessible energy region, typically up to $200 \mathrm{GeV}$, radiative corrections are dominated by the shift in the $W$ and $Z$ masses as parametrized by the $\rho$ parameter and by the running of the coupling constant. Vertex corrections and box diagrams involving gauge bosons are generally of minor importance. In the $\mathrm{TeV}$ region, accessible at future colliders like the LHC or TESLA, this picture changes drastically. A new class of effects starts to become relevant and rapidly dominant which are generally denoted as double logarithmic corrections and which were first observed by Sudakov [1] in the context of quantum electrodynamics for reactions with a tight cut on the radiated energy of the photons. For electroweak interactions large negative corrections arise from the exchange of gauge bosons which remain uncompensated if one restricts the analysis to exclusive final states, consisting e. g. of a fermion antifermion pair only. The discussion of double logarithmic corrections is fairly straightforward for a theory with massive gauge bosons only. An important complication arises from the presence of massless photons in the final state. Events with soft and hard photon

\footnotetext{
*Talk presented by J.H.Kühn

${ }^{\dagger}$ On leave from Institute for Nuclear Research of Russian Academy of Sciences, 117312 Moscow, Russia
}

radiation are normally included in the sample whence a "semiinclusive" definition of the cross section is closest to the the actual experimental analysis.

One loop corrections to the four fermion process are available since long (see e. g. [2]). In this case the separation of photonic and weak corrections is still possible and, employing axial gauge, the leading logarithms can be trivially attributed to fermion self energy diagrams with virtual $W$ or $Z$ boson emission [ 3].

Results for the leading logarithmic corrections in higher orders have been presented in [3, 田, 5, 6] (for a more recent discussion see also [ [8]). The first three results disagree, a consequence of different requirements on the inclusion or exclusion of hard photon emmission and a different treatment of the virtual corrections. In [ 6] it was demonstrated that the approach developed in [ 3], where axial gauge was adopted and both real and virtual photon emmission neglected, is numerically nearly identical to the results of [5] where virtual photon corrections and real photon emmission are included - a consequence of the smallness of the weak mixing angle, $\sin ^{2} \theta_{W}=0.23$. Furthermore, an analysis of subleading logarithms for the form factor, for the four fermion scattering in a spontaneously broken $S U(2)$ gauge theory and, last not least, for the Standard Model with $W, Z$ and the massless photon was performed in [6].

Before presenting a brief review of the techniques and some results from [ 6] let us emphasize the importance of subleading corrections. In the energy region relevant at LHC or TESLA, i.e. 
around 1 to $2 \mathrm{TEV}$, this is already evident from the one loop vertex correction. In the timelike region

$$
\begin{array}{r}
\left|F_{B}+\delta F\right|^{2} \approx F_{B}\left(1+2 \frac{\alpha_{W}}{\pi} \rho\left(s / M^{2}\right)\right), \\
\rho(u)=-\frac{1}{4} \ln ^{2} u+\frac{3}{4} \ln u-\frac{\pi^{2}}{12}+\frac{7}{8} .
\end{array}
$$

For characteristic values of the coupling, energy and mass of $\alpha_{W} / \pi=10^{-2}, C_{F}=3 / 4$ and $s / M^{2}=10^{2}$ one finds

$2 \frac{\alpha_{W}}{\pi} \rho=1.5 \times 10^{-2}(-5.30+3.45-1.70)=0.053$.

Large compensations between leading and subleading terms are observed and, in fact, this pattern will reappear for the Standard Model as discussed below.

The treatment of [ 6] is based on evolution equations that govern the dynamics of the amplitudes in the Sudakov limit as obtained in [ 9, 10, 11, 12, 13. In [6] this approach was applied to the next-to-leading analysis of the Abelian form factor and the four fermion amplitude in the $S U(N)$ gauge theory. Functions that enter the evolution equations in the next-to-leading logarithmic approximation were evaluated by using, as an input, asymptotic expansions of one-loop diagrams. The solution of these equations lead to a summation of the leading and subleading Sudakov logarithms. The expansion of one-loop diagrams through the so-called generalized strategy of regions [ 14] (see also [ 15]) identifies in a systematic way the nature of various contributions and the origin of logarithms. This strategy is based on expanding integrands of Feynman integrals in typical regions and extending the integration domains to the whole space of the loop momenta so that a crucial difference with respect to the standard approach [ $9,10,11,12,13]$ is the absence of cut-offs that specify the regions in individual terms of the expansions.

\section{The Abelian form factor in the Sudakov limit}

Let us first analyse the (vector) form factor which determines the amplitude of the fermion scattering in the external Abelian field. In Born approximation

$$
F_{B}=\bar{\psi}\left(p_{2}\right) \gamma_{\mu} \psi\left(p_{1}\right),
$$

We consider the limit $s=\left(p_{1}-p_{2}\right)^{2} \rightarrow-\infty$ with on-shell massless fermions, $p_{1}^{2}=p_{2}^{2}=0$, and gauge bosons with a small non-zero mass $M^{2} \ll$ $-s$. For convenience $p_{1,2}=(Q / 2,0,0, \mp Q / 2)$ so that $2 p_{1} p_{2}=Q^{2}=-s$.

The asymptotic behaviour can be found by solving the corresponding evolution equation [9]

$$
\begin{aligned}
& \frac{\partial}{\partial \ln Q^{2}} F= \\
& {\left[\int_{M^{2}}^{Q^{2}} \frac{\mathrm{d} x}{x} \gamma(\alpha(x))+\zeta\left(\alpha\left(Q^{2}\right)\right)+\xi\left(\alpha\left(M^{2}\right)\right)\right] F .}
\end{aligned}
$$

For the non-Abelian gauge theory, this equation was first derived in [ 10 by factorizing collinear logarithms in the axial gauge. Its solution is

$$
\begin{aligned}
& F=F_{0}\left(\alpha\left(M^{2}\right)\right) \exp \left\{\int_{M^{2}}^{Q^{2}} \frac{\mathrm{d} x}{x}\right. \\
& \left.\times\left[\int_{M^{2}}^{x} \frac{\mathrm{d} x^{\prime}}{x^{\prime}} \gamma\left(\alpha\left(x^{\prime}\right)\right)+\zeta(\alpha(x))+\xi\left(\alpha\left(M^{2}\right)\right)\right]\right\} .
\end{aligned}
$$

For a proper treatment of the next-to-leading logarithms one must keep renormalization group corrections to the leading logarithmic approximation as well as single infrared and renormalization group logarithms. In this approximation

$$
\begin{aligned}
F=F_{0}(\alpha) \exp [ & {\left[\int_{M^{2}}^{Q^{2}} \frac{\mathrm{d} x}{x} \int_{M^{2}}^{x} \frac{\mathrm{d} x^{\prime}}{x^{\prime}} \gamma\left(\alpha\left(x^{\prime}\right)\right)\right.} \\
& \left.+(\zeta(\alpha)+\xi(\alpha)) \ln \left(Q^{2} / M^{2}\right)\right]
\end{aligned}
$$

The leading terms of the functions $\gamma, \zeta$ and $\xi$ are obtained from the one loop analysis and the one loop running of $\alpha$ in the argument of the function $\gamma$ should be taken into account.

In the covariant gauge, the self energy insertions to the external fermion lines do not give $Q$-dependent contributions. The one loop calculation of the vertex correction gives

$$
F=\frac{\alpha}{2 \pi} C_{F}\left(-V_{0}+2 V_{1}+2(1-2 \epsilon) V_{2}-V_{2}^{\prime}\right) F_{B},
$$


where $C_{F}$ is the quadratic Casimir operator of the fundamental representation and the functions $V_{i}$ are obtained from

$$
\begin{aligned}
& \int \frac{\mathrm{d}^{d} k}{\left(k^{2}-2 p_{1} k\right)\left(k^{2}-2 p_{2} k\right)\left(k^{2}-M^{2}\right)}= \\
& \int \frac{i \pi^{d / 2} e^{-\gamma_{\mathrm{E}} \epsilon} s^{-1} V_{0}}{\left(k^{2}-2 p_{1} k\right)\left(k^{2}-2 p_{2} k\right)\left(k^{2}-M^{2}\right)}= \\
& \int \frac{i \pi^{d / 2} e^{-\gamma_{\mathrm{E}} \epsilon} s^{-1}\left(p_{1}+p_{2}\right)_{\mu} V_{1},}{\left(k^{2}-2 p_{1} k\right)\left(k^{2}-2 p_{2} k\right)\left(k^{2}-M^{2}\right)}= \\
& i \pi^{d / 2} e^{-\gamma_{\mathrm{E}} \epsilon}\left[g_{\mu \nu} V_{2}+\frac{p_{1 \mu} p_{2}+(\mu \leftrightarrow \nu)}{s} V_{2}^{\prime}\right]
\end{aligned}
$$

To expand these integrals in the limit $Q^{2} \gg$ $M^{2}$ we apply a generalized strategy of regions formulated in [14] and discussed using characteristic two-loop examples in [15]:

- Consider various regions of the loop momenta and expand, in every region, the integrand in Taylor series with respect to the parameters that are there considered small;

- Integrate the expanded integrand over the whole integration domain of the loop momenta;

- Put to zero any scaleless integral.

The following "typical" regions arise in the $\mathrm{Su}-$ dakov limit [16]:

$$
\begin{aligned}
\operatorname{hard}(\mathrm{h}): & k \sim Q \\
\text { 1-collinear (1c): } & k_{0}+k_{3} \sim Q \\
& k_{0}-k_{3} \sim M^{2} / Q, k_{1,2} \sim M \\
\text { 2-collinear (2c): } & k_{0}-k_{3} \sim Q \\
& k_{0}+k_{3} \sim M^{2} / Q, k_{1,2} \sim M \\
\text { soft (s): } & k \sim M \\
\text { ultrasoft (us): } & k \sim M^{2} / Q .
\end{aligned}
$$

Keeping the leading power in the expansion in the limit $Q^{2} / M^{2} \rightarrow \infty$ one observes [ [] that the leading double logarithm results from the hard region, whereas the single logarithm receives contributions from the hard and collinear regions as well. Soft regions do not contribute, at least in the leading power. Combining the vertex correction with the $Q^{2}$ independent fermion self energies one arrives at a finite result.

From the one-loop result one derives

$$
\gamma(\alpha)=-C_{F} \frac{\alpha}{2 \pi} .
$$

The total double logarithms originate from the hard region. This determines the scale of the coupling constant in the second order logarithmic derivative of the form factor in $Q$. At the same time we cannot distinguish, in the one loop approximation, the contribution to the functions $\zeta$ and $\xi$ coming from the collinear region because this region includes both $Q$ and $M$ scales. For the sum of these functions we find

$$
\zeta(\alpha)+\xi(\alpha)=3 C_{F} \frac{\alpha}{4 \pi} .
$$

Finally, in the NLO logarithmic approximation

$$
\begin{aligned}
F & =F_{B}\left(1-C_{F} \frac{\alpha}{2 \pi}\left(\frac{7}{2}+\frac{2 \pi^{2}}{3}\right)\right) \exp \left\{\frac{C_{F}}{2 \pi}[\right. \\
& \left.\left.-\int_{M^{2}}^{Q^{2}} \frac{\mathrm{d} x}{x} \int_{M^{2}}^{x} \frac{\mathrm{d} x^{\prime}}{x^{\prime}} \alpha\left(x^{\prime}\right)+3 \alpha \ln \left(Q^{2} / M^{2}\right)\right]\right\}
\end{aligned}
$$

in agreement with the result of [12, 13].

\section{The four fermion amplitude}

We study the limit of the fixed-angle scattering when all the invariant energy and momentum transfers of the process are much larger than the typical mass scale of internal particles $|s| \sim|t| \sim|u| \gg M^{2}$. Besides the extra kinematical variable the analysis of the four fermion amplitude is more complicated by the presence of different "color" and Lorentz structures. The Born amplitude, for example, can be expanded in the basis of color/chiral amplitudes

$$
\begin{array}{r}
A_{B}=\frac{i g^{2}}{s} A^{\lambda}=\frac{i g^{2}}{s} T_{F}\left(-\frac{1}{N}\left(A_{L L}^{d}+A_{L R}^{d}\right) \quad(11)\right. \\
\left.+A_{L L}^{c}+A_{L R}^{c}+(L \leftrightarrow R)\right),
\end{array}
$$


where

$$
\begin{aligned}
A^{\lambda} & =\bar{\psi}_{2}\left(p_{2}\right) t^{a} \gamma_{\mu} \psi_{1}\left(p_{1}\right) \bar{\psi}_{4}\left(p_{4}\right) t^{a} \gamma_{\mu} \psi_{3}\left(p_{3}\right) \\
A_{L L}^{d} & =\bar{\psi}_{2}^{i}{ }_{L} \gamma_{\mu} \psi_{1}{ }_{L}^{i} \bar{\psi}_{4}{ }^{j} \gamma_{\mu} \psi_{3}{ }_{L}^{j} \\
A_{L R}^{c} & =\bar{\psi}_{2}^{j}{ }_{L}^{j} \gamma_{\mu} \psi_{1}^{i}{ }_{L}^{i} \bar{\psi}_{4 R}^{i} \gamma_{\mu} \psi_{3}{ }_{R}^{j}
\end{aligned}
$$

and so on. Here $t^{a}$ is the $S U(N)$ generator, $p_{1}$, $p_{3}$ are incoming and $p_{2}, p_{4}$ outgoing momenta so that $t=\left(p_{1}-p_{4}\right)^{2}$ and $u=\left(p_{1}+p_{3}\right)^{2}=-(s+t)$. For the moment we consider a parity conserving theory. Hence only two chiral amplitudes are independent, for example, $L L$ and $L R$. Similarly only two color amplitudes are independent, for example, $A^{\lambda}$ and $A^{d}$.

Let us first compute the one loop corrections, employing again the strategy of regions. The total contribution from vertex and box diagrams in the logarithmic approximation is independent from chirality and the same both for the $L L$ and $L R$ amplitudes:

$$
\begin{array}{r}
\frac{i g^{2}\left(Q^{2}\right)}{s} \frac{1}{2}\left[\left\{-C_{F} L(s)+\left(3 C_{F}-C_{A} \ln \left(\frac{u}{s}\right)\right.\right.\right. \\
\left.\left.+2\left(C_{F}-\frac{T_{F}}{N}\right) \ln \left(\frac{u}{t}\right)\right) l(s)\right\} A^{\lambda} \\
\left.+\left\{2 \frac{C_{F} T_{F}}{N} \ln \left(\frac{u}{t}\right) \ln \left(\frac{-s}{M^{2}}\right)\right\} A^{d}\right]
\end{array}
$$

with

$L(s)=\frac{g^{2}}{16 \pi^{2}} \ln ^{2}\left(\frac{-s}{M^{2}}\right) ; l(s)=\frac{g^{2}}{16 \pi^{2}} \ln \left(\frac{-s}{M^{2}}\right)$ and the same both for the $L L$ and $L R$ amplitudes.

Now the collinear logarithms can be separated from the total one-loop correction. For each fermion-antifermion pair, they form the exponential factor found in the previous section (eq. (10)). This factor in addition incorporates the renormalization group logarithms which are not absorbed by changing the normalization scale of the gauge coupling. The rest of the single logarithms in eq. (13) is of the soft nature. Let us denote by $\tilde{A}$ the amplitude with the collinear logarithms factored out. It can be represented as a vector in the basis $A^{\lambda}, A^{d}$ and satisfies the following evolution equation [1], 17]

$$
\frac{\partial}{\partial \ln Q^{2}} \tilde{A}=\chi\left(\alpha\left(Q^{2}\right)\right) \tilde{A},
$$

where $\chi$ is the matrix of the "soft" anomalous dimensions. From eq. (14) we find the elements of this matrix to be, in units of $\alpha /(4 \pi)$,

$$
\begin{aligned}
& \chi_{\lambda \lambda}=-2 C_{A} \ln \left(\frac{u}{s}\right)+4\left(C_{F}-\frac{T_{F}}{N}\right) \ln \left(\frac{u}{t}\right) \\
& \chi_{\lambda d}=4 \frac{C_{F} T_{F}}{N} \ln \left(\frac{u}{t}\right) \\
& \chi_{d \lambda}=4 \ln \left(\frac{u}{t}\right) \\
& \chi_{d d}=0 .
\end{aligned}
$$

The solution of eq. (14) reads

$$
\begin{aligned}
\tilde{A}= & A_{1}^{0}\left(\alpha\left(M^{2}\right)\right) \exp \left[\int_{M^{2}}^{Q^{2}} \frac{\mathrm{d} x}{x} \chi_{1}(\alpha(x))\right] \\
& +A_{2}^{0}\left(\alpha\left(M^{2}\right)\right) \exp \left[\int_{M^{2}}^{Q^{2}} \frac{\mathrm{d} x}{x} \chi_{2}(\alpha(x))\right],
\end{aligned}
$$

where $\chi_{i}$ are eigenvalues of the $\chi$ matrix and $A_{i}^{0}$ are $Q$-independent vectors.

¿From the asymptotic expansion of the box diagrams one finds that only the hard parts contribute to eq. (14). This fixes the scale of $\alpha$ in this equation to be $Q$.

In the Abelian case, there are no different color amplitudes and there is only one anomalous dimension

$$
\chi=4 \ln \left(\frac{u}{t}\right) .
$$

\section{Sudakov logarithms in electroweak pro- cesses}

We are interested in the process $f^{\prime} \bar{f}^{\prime} \rightarrow f \bar{f}$. In the Born approximation, its amplitude is of the following form [3]

$$
A_{B}=\frac{i g^{2}}{s} \sum_{I, J=L, R}\left(T_{f^{\prime}}^{3} T_{f}^{3}+t_{W}^{2} \frac{Y_{f^{\prime}} Y_{f}}{4}\right) A_{I J}^{f^{\prime} f}
$$

where

$$
A_{I J}^{f^{\prime} f}=\bar{f}_{I}^{\prime} \gamma_{\mu} f_{I}^{\prime} \bar{f}_{J} \gamma_{\mu} f_{J}
$$

To analyze the electroweak correction to the above process we use the approximation with the $W$ and $Z$ bosons of the same mass $M$ and massless quarks and leptons. The photon is massless, 
and the corresponding infrared divergent contributions should be accompanied by the real soft photon radiation integrated to some resolution energy $\omega_{\text {res }}$ to get an infrared safe cross section independent on an auxiliary photon mass. At the same time the massive gauge bosons are supposed to be detected as separate particles. In practice, the resolution energy is much less than the $W$ $(Z)$ boson mass so the soft photon emission is of the QED nature. This cancels the infrared singularities of the QED virtual correction. We should therefore separate the QED virtual correction from the complete result computed with the photon of some mass $\lambda$ and then evaluate the QED virtual corrections together with the real soft photon radiation effects with vanishing $\lambda$. It is convenient to subtract the QED contribution computed with the photon of the mass $M$ from the obtained result for the virtual corrections and then take the limit $\lambda \rightarrow 0$ for the sum of QED virtual and real photon contributions to the total amplitude. In the language of the approach of ref. [5], this prescription means that we use the auxiliary photon mass $\lambda$ as a variable of the evolution equation below the scale $M$ and the subtraction fixes a relevant initial condition for this differential equation. This leads to a modification of the collinear factor and the soft anomalous dimensions.

Thus we keep always a cutoff $\omega_{\text {res }} \ll M$ and do not display the QED Sudakov factor arising from this suppression of photon radiation. The remaining "electroweak" universal collinear factor for each fermion-antifermion pair becomes

$$
\begin{array}{r}
\exp \left[-\left(T_{f}\left(T_{f}+1\right)+t_{W}^{2} \frac{Y_{f}^{2}}{4}-s_{W}^{2} Q_{f}^{2}\right)\right. \\
\times(L(s)-3 l(s))] .
\end{array}
$$

The soft anomalous dimension for $I$ and/or $J=$ $R$ is Abelian and, in units of $g^{2} /\left(16 \pi^{2}\right)$, reads

$$
\chi=\left(t_{W}^{2} Y_{f^{\prime}} Y_{f}-4 s_{W}^{2} Q_{f^{\prime}} Q_{f}\right) \ln \left(\frac{u}{t}\right),
$$

and the matrix of the soft anomalous dimension for $I=J=L$ is

$$
\begin{aligned}
\chi_{\lambda \lambda}= & -4 \ln \left(\frac{u}{s}\right) \\
& +\left(t_{W}^{2} Y_{f^{\prime}} Y_{f}-4 s_{W}^{2} Q_{f^{\prime}} Q_{f}+2\right) \ln \left(\frac{u}{t}\right) \\
\chi_{\lambda d}= & \frac{3}{4} \ln \left(\frac{u}{t}\right) \\
\chi_{d \lambda}= & 4 \ln \left(\frac{u}{t}\right) \\
\chi_{d d}= & \left(t_{W}^{2} Y_{f^{\prime}} Y_{f}-4 s_{W}^{2} Q_{f^{\prime}} Q_{f}\right) \ln \left(\frac{u}{t}\right) .
\end{aligned}
$$

The one-loop leading and subleading logarithms can be directly obtained from eq. (13). The twoloop leading (infrared) logarithms are determined by the second order term of the expansion of the double (soft $\times$ collinear) logarithmic part of the collinear factors 200. The two-loop nextto-leading logarithms are generated by the interference between the first order terms of the expansion of the double (soft $\times$ collinear) and single (soft+collinear+renormalization group) logarithmic exponents and can also be found in [ 6. With the expression for the chiral amplitudes at hand, we can compute the leading and subleading logarithmic corrections to the basic observables for $e^{+} e^{-} \rightarrow f \bar{f}$. Let us, for example, consider the total cross sections of the quarkantiquark $/ \mu^{+} \mu^{-}$production in the $e^{+} e^{-}$annihilation. In the two loop approximation, the leading and next-to-leading Sudakov corrections to the cross sections read

$$
\begin{aligned}
\sigma / \sigma_{B}\left(e^{+} e^{-} \rightarrow Q \bar{Q}\right) & =1+5.30 l(s)-1.66 L(s) \\
- & 12.84 l(s) L(s)+1.92 L^{2}(s), \\
\sigma / \sigma_{B}\left(e^{+} e^{-} \rightarrow q \bar{q}\right) & =1+20.54 l(s)-2.17 L(s) \\
- & 53.72 l(s) L(s)+2.79 L^{2}(s), \\
\sigma / \sigma_{B}\left(e^{+} e^{-} \rightarrow \mu^{+} \mu^{-}\right) & =1+10.09 l(s)-1.39 L(s) \\
- & 21.66 l(s) L(s)+1.41 L^{2}(s),
\end{aligned}
$$

where $Q=u, c, t, q=d, s, b$. Numerically, $L(s)=$ $0.07(0.11)$ and $l(s)=0.014(0.017)$ respectively for $\sqrt{s}=1 \mathrm{TeV}$ and $2 \mathrm{TeV}$.

Clearly, for energies at 1 and $2 \mathrm{TeV}$ the two loop corrections are huge and amount up respectively to $5 \%$ and $7 \%$. There is a cancellation 
between the leading and subleading logarithms and for the above energy interval the subleading contribution even exceeds the leading one. The higher order leading and next-to-leading corrections however do not exceed $1 \%$ level. They can be in principle resummed using the formulae given above. The leading and subleading corrections to the left right and forward backward asymmetries [6] are typically smaller.

Our result for the one loop double logarithmic contribution is in agreement with [ [t]. However the result for the one loop single infrared logarithmic contribution differs from [18]. The reason is that, in [18], only the diagrams with heavy virtual bosons have been taken into account. There is an infrared safe contribution of the diagram with the virtual massless photon where the heavy boson mass serves as an infrared regulator that should be taken into account to get a complete (exponential) result. In one-loop approximation, this contribution comes from the box diagrams with the photon and $Z$ boson running inside the loop [19].

Our result for the two-loop double logarithmic contribution is in agreement with [5]. On the other hand, the coefficients in front of the twoloop leading logarithms in eq. (23) with a few percent accuracy coincide with the result of [ [3] where the photon contributions were not considered. This is related to the fact that the virtual photon contribution not included to the result of [3] is suppressed by a small factor $s_{W}^{2}$.

\section{Acknowledgments}

The work by V.S. was supported by the Volkswagen Foundation, contract No. I/73611. The work by J.K. and A.P. was supported by the Volkswagen Foundation, by BMBF under grant BMBF057KA92P and by DFG-Forschergruppe "Quantenfeldtheorie, Computeralgebra und MonteCarlo-Simulationen" (DFG Contract KU502/6$1)$.

\section{REFERENCES}

1. V.V. Sudakov, Zh. Eksp. Teor. Fiz. 30 (1956) 87.
2. W. Beenakker, W. Hollik and Van der Mark, Nucl. Phys. B365 (1991) 24.

3. J.H. Kühn and A.A. Penin, Preprint TTP/99-28, hep-ph/9906545.

4. P. Ciafaloni and D. Comelli, Phys. Lett. B446 (1999) 278.

5. V.S. Fadin, L.N. Lipatov, A.D. Martin and M. Melles, Preprint PSI PR-99-24, hepph/9910338.

6. J.H. Kühn, A.A. Penin and V.A. Smirnov, Preprint DESY/00-008, TTP/99-54, hepph/9912503.

7. M. Melles, Preprint PSI PR-00-07, hep$\mathrm{ph} / 0004056$.

8. W. Beenakker, these proceedings.

9. J.C. Collins, Phys.Rev. D22 (1980) 1478; in Perturbative QCD, ed. A.H. Mueller, 1989, p. 573.

10. A. Sen, Phys. Rev. D24 (1981) 3281.

11. A. Sen, Phys. Rev. D28 (1983) 860.

12. G. Korchemsky, Phys. Lett. B217 (1989) 330.

13. G. Korchemsky, Phys. Lett. B220 (1989) 629. D42 (1990) 4222.

14. M. Beneke and V.A. Smirnov, Nucl. Phys. B522 (1998) 321.

15. V.A. Smirnov and E.R. Rakhmetov, Teor. Mat. Fiz. 120 (1999) 64; V.A. Smirnov, Phys. Lett. B465 (1999) 226.

16. G. Sterman Phys. Rev. D17 (1978) 2773;

S.Libby and G. Sterman Phys. Rev. D18 (1978) 3252;

A.H. Mueller, Phys. Rep. 73 (1981) 35.

17. J. Botts and G. Sterman, Nucl. Phys. B325 (1989) 62;

N. Kidonakis, G Odereda and G. Sterman, Nucl. Phys. B531 (1998) 365.

18. M. Beccaria et al., Preprint PM/99-26, hepph/9906319.

19. J.H. Kühn and R.G. Stuart, Phys. Lett. B200 (1987) 360. 